\section{The Influence of TEM Education on TEM Development}

\section{B. Williams, Lehigh University}

Learning how to operate the microscope has dominated the TEMteaching process in a way that is perhaps unparalleled by any other common scientific instrument. The TEM column is so complex that the arcane aspects of its manual manipulation become the main points to be learned by the student, rather than the use of the instrument to solve materials problems. In other words, command of the instrument often becomes an end in itself. Such an approach would preach that it is better to be able to produce a striking CDF image that is well aligned and focused than it is to be able to interpret that image in terms of the materials processing responsible for the microstructure!

The teaching of TEM has taken two different approaches depending on the background of the author(s) of the textbooks used in the classroom:

a) A theoretical metal-physics approach: e.g., Hirsch, Howie, Nicholson, Pashley \& Whelan (HHNPW). (1965, 1977), Reimer (1984 and several subsequent editions)

b) A more practical metallurgy/materials approach: e.g., Thomas, (1962), Edington (1976)

The metal-physics approach stresses the physics behind beamspecimen interactions and interpretation of the images and diffraction patterns. This occupies $\sim 450$ pages in HHNPW. Interpretation is generally in terms of understanding the theories of image/DP formation, not the processing that gave rise to the structure, or the properties that are imparted to the material by the structure. Such physics-based texts are short on specimenpreparation information. HHPNW has 26 pages. Similarly, Reimer (1984) devotes 0 pages (out of $>500$ ) to specimen preparation. One principle of this general approach to TEM teaching seems to be 'never use a few lines of text when a differential equation will do'. While such texts are invaluable to accomplished microscopists, they are daunting to most starting students.

The metallurgical approach is typified by the textbooks of Thomas (1963) and Edington (1976), which emphasize the TEM as a tool for solving materials problems. Therefore, this approach stresses the need for careful specimen preparation as the crucial step in any TEM investigation. (Thomas has 54 out of 263 pages and Edington 140 out of 350 pages, respectively). In these texts, interpretation of images and diffraction patterns are emphasized primarily in terms of their relation to materials science and engineering, rather than understanding the mathematics of image and diffraction theory.

Of the two approaches, I believe it metal-physics has dominated the field, perhaps not in terms of the numbers of students produced, but in terms of the emphasis on the instrument itself, that still pervades the TEM classroom. When the instrument performance alone is the focus of the teaching (which it has to be given the complexity of the column) then such aspects as pushing the image resolution become ingrained in the student. When that student becomes a professional charged with purchasing another TEM, resolution becomes the foremost requirement of the instrument, and such factors have dominated its development. Over the years this somewhat circular argument has resulted in serious investment in expensive in high-voltage, highresolution, and atomic-resolution microscopes. While not denying the important role such high-end instruments have to play in materials science, they are not needed to address most of the problems for which the TEM is well designed to solve, so they should not be the prime drivers of the TEM industry.

Consequently, it is my belief that for most of the 60 years of its commercial development, the TEM has been designed for the small fraction of operators who seek to push the resolution envelope. The goal of $1 \AA$ resolution in TEM images has been pursued with great vigor and expense over the last decade, because that simply means that the instrument is getting "better", rather than the fact that more problems will be solved more easily. Phasecontrast image resolution is still the single most important performance specification when TEMs are purchased, despite the irrelevance of that criterion to most TEM studies. Almost all TEMs sold to materials users today are capable of some degree of atomic resolution $(<2.5 \AA)$ and, as a result, carry a price tag $>\$ 1 \mathrm{M}$. There are few "run of the mill" TEMs sold, and certainly none available with a price tag of less than $\$ 250,000$, typical of a high-end SEM.

The almost universal demand for atomic-resolution capabilities in new TEMs ignores the fact that most TEM users never produce an image close to the resolution limit of their TEM. This is because:

a) The specimen isn't thin enough, chromatic aberration limits resolution significantly worse than spherical-aberration limits.

b) Even if a phase-contrast structure image is produced, the user doesn't understand the theory or doesn't have the computer skills/software to simulate the images in the way required for correct interpretation.

It is worth contrasting the history of TEM education with that of the SEM. The SEM has generally been looked upon as a poor relation of the TEM. "Real microscopists don't use SEM," said David Joy, sarcastically, at the Cranbrook meeting in 1984. Such a view, however, was unfortunately true amongst many TEM users and resulted in the exclusion of the SEM from EMSA meetings for many years and the generation of many competitive microscopy meetings, diluting the field. There is no kudos to be gained by mastering the SEM, in contrast to the TEM. This view has arisen, in my opinion, because the strengths of SEM are at lower magnification where the depth of field becomes a tremendous asset, so it isn't a "real microscope". Consequently the primary aim of SEM education has never been on teaching the electron optics or pushing the resolution limit, but more on optimizing the instrument to obtain the best information possible. (For example, combining EDS with imaging is well optimized in most SEMs, but after more than 25 years the EDS generally has a non-optimal interface in most analytical TEMs.). Because the SEM has been developed with the user in mind, over the last decade it has been transformed into a fully digitized, computer-controlled instrument, operable by almost anyone with a high-school education, after a few hours of training. At the same time, the SEM has undergone a revolution in experimental capabilities and, in concert with the advent of this user-friendly design, it has developed to the point where image resolution routinely approaches that of chromatic-aberration limited TEM (l.e., $<2 \mathrm{~nm})$. Furthermore, its diffraction capabilities via electron backscatter patterns (EBSP), approach that of CBED in the TEM - except that the EBSP analysis is fully computer controlled, allowing (with help from EDS) phase identification, on line, in a matter of a few minutes. This specific task has theoretically been within the capabilities of TEM for more than 20 year but has not been commercially implemented in any widespread manner.

The trend towards user-friendliness is aided in part by the significantly greater degree of competition in the SEM than in the TEM marketplace, and the dominance of that market by industrial users rather than users in university and research laboratories. Since semiconductor manufacturers buy SEMs by the dozen, the manufacturers have responded rapidly to the needs of that specific industry. In particular the semiconductor companies have pushed for SEMs that can be quickly installed anywhere, and perform to their limits with an operator who only the previous week was uncrating the SEM on the loading dock. Consequently, SEM designers have emphasized aspects such as the ease of operation (e.g., the ability to take many pictures, all in focus), rather than ultimate lens performance. The users' needs are paramount and there are more users in industry than in academe. Industrial users are less skilled in microscope operation than users in academe.

Despite such 'unreasonable' performance demands by the prime users over the last decade, the SEM has become a user-friendly routine scientific instrument. TEMs should be as easy to install and operate. However, each TEM is designed primarily to manufacturers' specifications rather than users' specifications. A TEM will only operate to its resolution limits in a room that costs almost 
as much as a high-end SEM and, because it costs so much, any TEM must always be sold as a top of the line instrument. Part of the reason for this is that the TEM market is not controlled by any one user-group. We all have different specifications for our own instruments, and we order them one at a time. Because we invest $\$ 1 \mathrm{M}$ or more, we somehow don't feel it is unreasonable to spend hundreds of thousands of dollars in addition to provide an environment in which our TEM can deliver its atomic resolution. Perhaps we should be more demanding.

As a result of the emphasis on TEM resolution above all else, the market for TEMs has generally decreased over the last ten years or so to the point where there are now fewer manufacturers and higher prices than ever before. This situation is dangerous. If the market continues to stagnate or contract further and prices of TEMs remain high in comparison to other sophisticated characterization tools, then it may not be long before we see even fewer manufacturers and fewer choices. To avoid this, I believe that users and manufacturers must work together to design the next generation of TEMs. A large fraction of the next generation of TEMs must be fully computer controlled, simple to operate with the average user in mind and, above all, priced at a level such that routine users may be able to consider a purchase. One aspect of such an instrument should be that it is operable remotely over the Internet, since that would require complete computer control for easy operation.

\section{References:}

Hirsch, P.B., Howie, A. Nicholson, R.B., Pashley, D. W and Whelan, M.J. (1963) Electron Microscopy of Thin Crystals, Butterworths, London; (second edition, 1977), Krieger. NY

Reimer, L. (1984) Transmission Electron Microscopy, (1st edition) Springer Ver$\operatorname{lag}, N Y$

Edington, J. W. (1976) Practical Electron Microscopy in Materials Science, Van Nostrand Reinhold, NY
Thomas, G. (1962) Transmission Electron Microscopy, John Wiley \& Sons, NY This article is based on a talk given at a symposium on "The Future of Remote Microscopy in Materials Education" at Carnegie-Mellon University, October 25, 1998

Ed. Note: Dr. Williams acknowledges that the above may be considered a bit "provocative" by some, and comments are more than welcome.

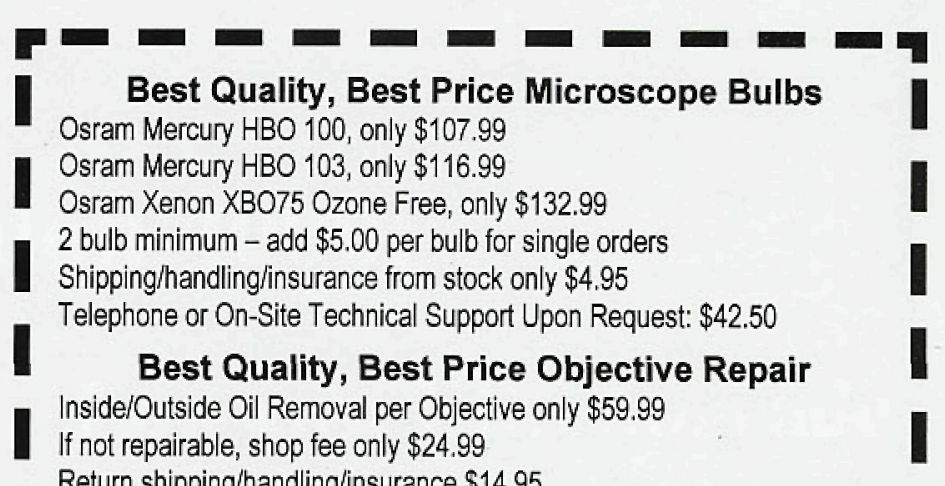

Return shipping/handling/insurance $\$ 14.95$

\section{Sciscope Instrument Company Leica Kodak Sony Osram Dealer \\ Mail orders: Box 2237, lowa City, lowa 52244 USA USA telephone orders: 1-800-55-MICRO \\ Overseas telephone orders: +319-338-1107}

Fax orders: 1-319-338-3954 Internet orders: www.sciscope.com

* Include your Visa, Master Card or American Express information

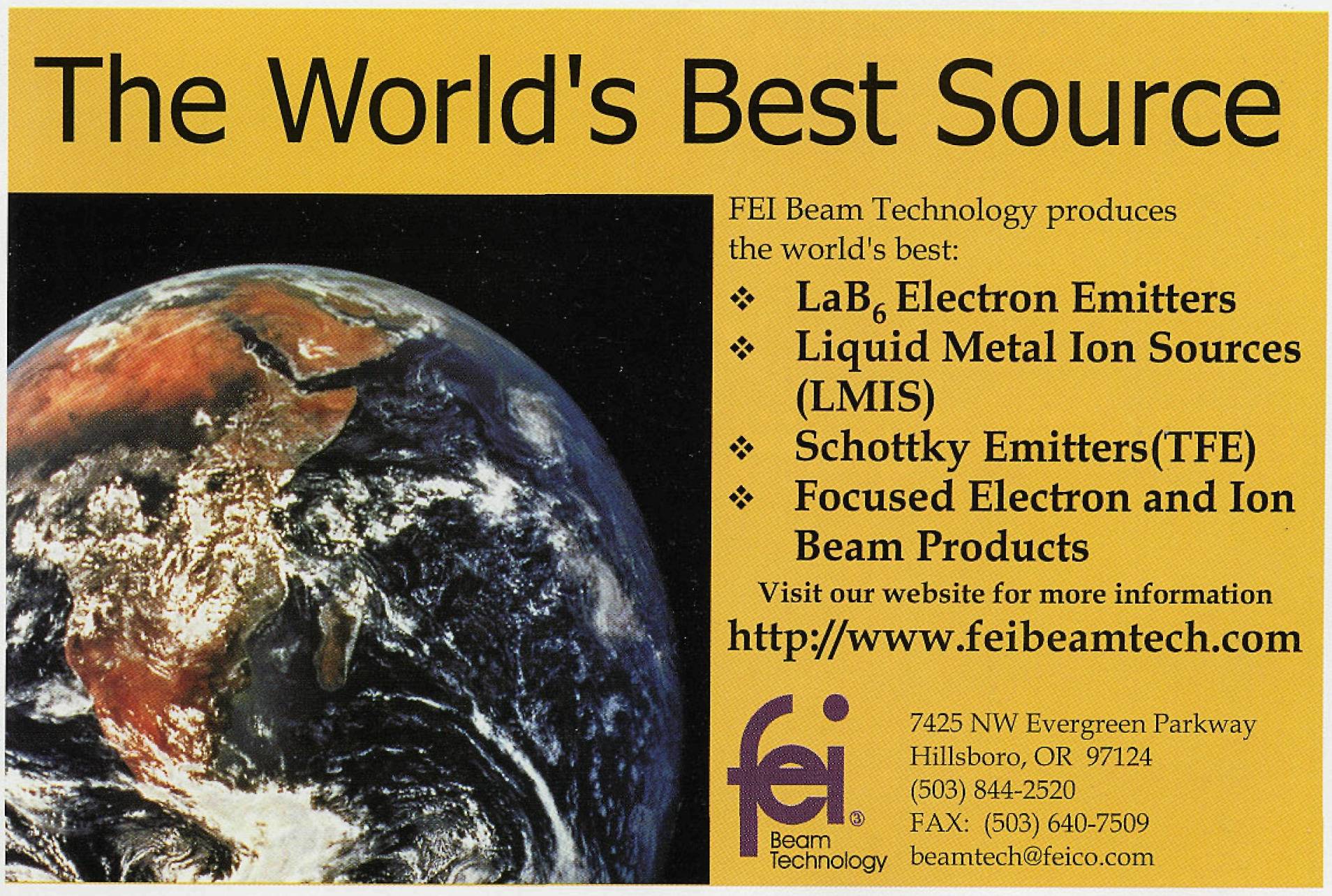

